Current Research in Dentistry 1 (2): 23-28, 2010

ISSN 1949-0119

(C) 2010 Science Publications

\title{
Effect of Saliva Moistening on Shear Bond Strength of Self Ligating Orthodontic Brackets
}

\author{
Maria Francesca Sfondrini, Danilo Fraticelli, \\ Benedetta Balconi, Andrea Scribante and Paola Gandini \\ Department of Orthodontics, Faculty of Dentistry, University of Pavia, Italy
}

\begin{abstract}
Problem statement: The purpose of the present study was to compare Shear Bond Strength (SBS) and bond failure site of conventional and Self-Ligating (SL) orthodontic brackets, bonded on dry and saliva contaminated enamel. Approach: One conventional and three different types of self ligating stainless steel brackets were bonded onto 160 bovine permanent mandibular incisors, divided randomly into 8 groups, using Transbond XT adhesive system. For each type of bracket, 20 samples were bonded on dry enamel and 20 after saliva contamination. After $24 \mathrm{~h}$ all specimens were tested for SBS using an instron universal testing machine and Adhesive Remnant Index (ARI) were evaluated. Results: Saliva contamination significantly lowered SBS of conventional brackets. For self-ligating brackets no significant strength reduction was recorded. Conclusion: All the groups showed clinically adequate mean shear bond strengths. ARI scores in contaminated groups resulted lower than in dry groups.
\end{abstract}

Key words: Self-Ligating (SL), Shear Bond Strength (SBS), Adhesive Remnant Index (ARI), contaminated groups resulted, frequent encountered contamination

\section{INTRODUCTION}

Despite the continuous progresses in direct bonding technique, the brackets unexpected detachment is one of the biggest problems orthodontists have to face. In fact one of the parameter should be considered inchoosing restorative material is its shear bond strength (Farrokhgisu and Eskandarizade, 2010). For clinical success, in fact, brackets must resist a displacement force of at least $515 \mathrm{~kg}$ because the attachment must allow the delivery of orthodontic forces and has to withstand masticatory loads. In addition, the bracket must be easily removed at the end of treatment, resulting in minimal hard and soft tissue damages during application, service and removal (Knox et al., 2000).

A critical factor that affects bond strength of adhesion is wetness due to water, saliva or blood contamination of the etched enamel surface. Contamination during the adhesive application reduces the longevity of the bonding materials, once it can alter the material properties, substrate surface and causes problems in the bonding process (Retamoso et al., 2009). However, various clinical conditions do not permit ideal isolation of the bonding site, especially when bonding attachments in hard-to-reach places near gingival area, around second molar, when exposing and attaching buttons to partially erupted or impacted ectopic teeth (Oztoprak et al., 2007). When etched enamel becomes wet, most of the porosities become plugged and resin penetration is impaired, resulting in resin tags of insufficient number and length; when contamination is produced after the primer application, it is not only the hydrophilic capacity of the primer that is important, but also the bracket bonding resin (Vicente et al., 2009). Water contamination is more easily avoided than saliva or blood contamination because these two can deposit an organic adhesive coating within the first few $\mathrm{sec}$ of exposure that is resistant to washing (Sfondrini et al., 2004). Moreover, saliva is reported to be the most frequent encountered contamination in the clinic and even momentary saliva contact adversely affects the bond (Sfondrini et al., 2004).

Concerning with the moment in which contamination occurs, the literature presents different reports. Some Authors (Paschos et al., 2008; Faltermeier et al., 2007) pointed out that even single saliva contamination (before or after priming) can cause a significant reduction in SBS, but this doesn't happen when enamel is reprimed after the contamination (Webster et al., 2001). (Retamoso et al., 2009), instead, concluded that saliva contamination before priming has only a little influence on SBS. Finally, another study (Cacciafesta et al., 2003) revealed a significant decrease in SBS, till clinically unacceptable values, only when contamination is introduced after primer polymerization.

To date, in literature, there are not studies that compare SBS of self-ligating brackets in presence of saliva contamination.

Corresponding Author: Andrea Scribante, Department of Orthodontics, Faculty of Dentistry, University of Pavia, Italy 
The aim of the present study was to test three different self-ligating brackets bonded on dry and saliva contaminated enamel. As far as saliva contamination before primer application is easily avoidable, because the presence of moisture on chalky white enamel is readily identifiable, saliva was applied after primer polymerization. Shear bond strengths and Adhesive Remnant Index (ARI) have been evaluated and compared with those of a conventional orthodontic bracket.

\section{MATERIALS AND METHODS}

One hundred and sixty freshly extracted bovine permanent mandibular incisors were collected from a local slaughterhouse and stored in a solution of $0.1 \%$ (weight/volume) thymol. The criteria for tooth selection included intact buccal enamel with no cracks caused by extraction and no caries. The teeth were cleansed of soft tissues and embedded in cold-curing, fast-setting acrylic (Leocryl, Leone, Sesto Fiorentino, Italy). Metal rings (15 mm-diameter) were filled with the acrylic resin and allowed to cure, thus encasing each specimen while allowing the buccal surface of enamel to be exposed. Each tooth was oriented so that the labial surface was parallel to the shearing force.

The teeth were then divided randomly into 8 groups of 20 specimens. Four different orthodontic stainless steel maxillary central incisor brackets were tested: 40 Step (Leone, Sesto Fiorentino, Italy), 40 SL Damon (Ormco, Glendora, California, USA), 40 SL Smart Clip (3M Unitek, Monrovia, California, USA), 40 SL Quick (Forestadent, Pforzheim, Germany).

Before bonding, the labial surface of each incisor was cleaned for $10 \mathrm{sec}$ with a mixture of water and fluoride-free pumice in a rubber-polishing cup with a slow-speed handpiece. The enamel surface was rinsed with water to remove pumice or debris and then dried with an oil-free air stream.

The teeth were etched with $37 \%$ phosphoric acid gel (3M Unitek, Monrovia, California, USA) for $30 \mathrm{sec}$, followed by thorough washing for $20 \mathrm{sec}$ and drying for other $20 \mathrm{sec}$. A thin layer of primer (Transbond XT, 3M Unitek, Monrovia, California, USA) was applied to the etched enamel, stretched out with an oil-free air stream and light-cured for $20 \mathrm{sec}$ at $1 \mathrm{~mm}$-distance with a led light-curing unit (Mectron Starlight Pro, Dental 200, Mantova, Italia). At this stage, in contaminated groups (Knox et al., 2000; Oztoprak et al., 2007; Sfondrini et al., 2004; Paschos et al., 2008), a thin layer of human saliva from one female donator, was applied with a brush, without including air bubbles between saliva and tooth primed surface. Then, all brackets were bonded with composite resin (Transbond XT, 3M Unitek,
Monrovia, California, USA) near the centre of the facial surface of the teeth. Sufficient pressure was applied to express excess adhesive, which was removed from the margins of the bracket base with a scaler before polymerisation. The brackets were then light cured with a visible light-curing unit (Mectron Starlight Pro, Dental 200, Mantova, Italia) for $10 \mathrm{sec}$ on the mesial and $10 \mathrm{sec}$ on the distal side (total cure time $20 \mathrm{sec}$ ).

After bonding, all samples were stored in distilled water at room temperature for $24 \mathrm{~h}$ and then tested in shear mode on an Instron Universal Testing Machine 3343 (Instron Industrial Products, Grove City, Pennsylvania, USA). The specimens were secured in the lower jaw of the machine so that the bonded bracket base was parallel to the direction of the shearing force. The specimens were stressed in an occluso-gingival direction at a crosshead speed of $1 \mathrm{~mm} \mathrm{~min}^{-1}$, as in previous studies (Cacciafesta et al., 2003; Jobalia et al., 1997; Millett et al., 1999; Sfondrini et al., 2011). The maximum load necessary to debond or initiate bracket failure was recorded in Newtons and then converted into megaPascals as a ratio of force to surface area of the bracket $\left(\mathrm{MPa}=\mathrm{N} / \mathrm{mm}^{2}\right)$.

After bond failure, the bracket bases and the enamel surfaces were examined and Adhesive Remnant Index was used to assess the amount of adhesive left on the enamel surface. This scale ranges from 0-3. A score of 0 indicates no adhesive remaining on the tooth; 1 means less than half of the adhesive left on tooth surface; 2 denotes more than half of the adhesive remaining on the tooth and 3 stands for all adhesive still on the tooth surface, with a distinct impression of the mesh base. The ARI scores have been used as a more complex method for defining bond failure site among: enamel-adhesive interface, within the composite resin and bracket baseadhesive interface (Artun and Bergland, 1984).

Statistical analysis was performed with Stata 7.0 software (Stata, College Station, Texas, USA). Descriptive statistics, including the mean, standard deviation, median, minimum and maximum values were calculated for all groups. Analysis of Variance (ANOVA) was applied to determine whether significant differences in debond strength values existed among the groups. Tukey test was used for post hoc comparison. The chi-square test was used to determine significant differences in the ARI scores among the different groups. Significance for all statistical tests was predetermined at $\mathrm{p}<0.05$.

\section{RESULTS}

Descriptive statistics for the SBS (MPa) of the different groups are illustrated in Table 1 and Fig. 1. 
Table 1: Descriptive statistics of the different groups (*: groups with the same letters are not significantly different)

\begin{tabular}{lllllllll}
\hline Group & Bracket & Surface & Mean & SD & Min & Mdn & Max & Tukey* \\
\hline 1 & Step & Dry & 13,71 & 3,43 & 5,55 & 13,45 & 20,96 & A \\
2 & Step & Saliva & 8,81 & 3,65 & 3,6 & 7,7 & 16,76 & B \\
3 & Smart clip & Dry & 13,97 & 5,01 & 5,96 & 11,86 & 24,69 & A \\
4 & Smart clip & Saliva & 12,3 & 3,59 & 8,19 & 11,1 & 19,68 & A \\
5 & Quick & Dry & 19,67 & 6,27 & 6,61 & 18,55 & 34,51 & C \\
6 & Quick & Saliva & 18,82 & 5,7 & 3,9 & 20,49 & 32,65 & C \\
7 & Damon & Dry & 8,82 & 2,24 & 4,69 & 8,66 & 14 & B \\
8 & Damon & Saliva & 6,7 & 1,63 & 3,55 & 6,94 & 9,56 & B \\
\hline
\end{tabular}

Table 2: ARI scores of the different groups

\begin{tabular}{lllllll}
\hline Group & Bracket & Surface & ARI =0 (\%) ARI = 1(\%) & ARI =2 (\%) & ARI = 3 (\%) \\
\hline 1 & Step & Dry & $2(10)$ & $1(5)$ & $10(50)$ & $7(35)$ \\
2 & Step & Saliva & $0(0)$ & $2(10)$ & $10(50)$ & $8(40)$ \\
3 & Smart Clip & Dry & $0(0)$ & $5(25)$ & $9(45)$ & $6(30)$ \\
4 & Smart Clip & Saliva & $1(5)$ & $8(40)$ & $10(50)$ & $1(5)$ \\
5 & Quick & Dry & $0(0)$ & $2(10)$ & $7(35)$ & $11(55)$ \\
6 & Quick & Saliva & $0(0)$ & $2(10)$ & $10(50)$ & $8(40)$ \\
7 & Damon & Dry & $4(20)$ & $3(15)$ & $3(15)$ & $10(50)$ \\
8 & Damon & Saliva & $1(5)$ & $9(45)$ & $9(45)$ & $1(5)$ \\
\hline
\end{tabular}

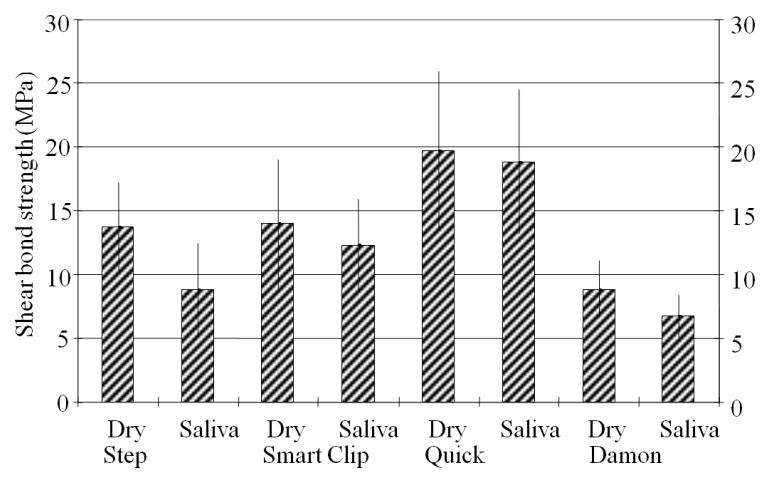

Fig. 1: Mean shear bond strength of the 8 different groups

Normality of data was calculated using the Kolmogorov-Smirnov test. ANOVA showed the presence of significant differences among the various groups $(\mathrm{p}<0.001)$. Tukey post-hoc test showed significant differences between SBSs of group 1 and 2 (conventional brackets). No significant differences were found between dry and saliva groups for self-ligating brackets.Both on dry and contaminated enamel, Quick SL brackets demonstrated the highest SBSs, compared with all other groups. Besides, SL Smart Clip brackets recorded significantly higher SBSs than SL Damon, both in dry and saliva groups.

The results of ARI scores are presented in Table 2. Frequency analysis of ARI scores showed significant higher frequency of ARI "2" for groups 1,2,3,4 (Step and Smart Clip). A significant reduction of ARI scores has been found for SL Quick and Damon brackets. For Quick SL brackets, in dry group (5) there was a prevalence of score " 3 " that decreased to " 2 " in saliva group (6); while for Damon brackets, ARI score decreased from "3" with dry enamel (group 7) to a preponderance of " 1 " and " 2 " when contamination occurred (group 8).

\section{DISCUSSION}

Many studies have been conducted to improve brackets adhesion to enamel surfaces, working on the different variables that concur in bonding success: bracket base design (Sharma-Sayal et al., 2003; Wang et al., 2004; Merone et al., 2010), surface treatments (Sharma-Sayal et al., 2003; Wright and Powers, 1985; Newman et al., 1995; Basudan and Al-Emran, 2001; Tavares et al., 2006); bracket materials (Bishara et al., 2002); enamel conditioning agents and acid concentration (Wang et al., 2004; Bishara et al., 1998; Cacciafesta et al., 2004; Wang and Lu, 1991).

Reynolds (1975) defined the minimum value of bond strength that was necessary to withstand clinical forces for orthodontic brackets: 6-8 MPa. In the present study, mean SBS recorded for each type of bracket was over that value, assuring the possibility of clinical use for all the attachment tested. However, there were a lot of variations in the values registered in the different groups.

In literature, bonding contamination by saliva has been studied, in vivo and in vitro, for conventional brackets, evaluating SBS on dentin and enamel (Townsend and Dunn, 2004), recording a significant reduction in bond strength. Some studies (Retamoso et al., 2009; Paschos et al., 2008; Webster et al., 2001) showed a significant reduction in SBS in presence of saliva, however the values they collected where sufficient to withstand clinical forces. Other Authors (Faltermeier et al., 2007; Cacciafesta et al., 2003) instead, in case of saliva contamination obtained clinically unacceptable values.

Concerning with conventional brackets Step, SBS recorded for dry group (group 1) were similar to those of previous studies (Sfondrini et al., 2011; Northrup et al., 2007) and significantly higher than SBSs of saliva specimens (group 2) $(\mathrm{p}<0.05)$.

There are only two published studies in the literature relating to the SBS of self-ligating brackets on dry enamel. Sfondrini et al. (2011) compared the SBS of conventional brackets with those of three different self-ligating brackets: self-ligating Smart Clip (3M Unitek) and Damon 3MX (Ormco) brackets showed significantly higher SBS values than conventional Step (Leone) and self-ligating Quick (Forestadent) brackets. The latter (Northrup et al., 2007), instead, compares the SBS of a conventional and a self-ligating bracket using two different bonding systems (Transbond XT, 3M Unitek; Orthosolo, Ormco): despite of a statistically significant difference in bond strength between the 
conventional Orthos (Ormco) bracket group and both selfligating Damon 2 (Ormco) brackets, all three groups produced clinically acceptable mean SBSs in vitro.

The results obtained by Northrup et al. (2007) are in contrast with those of the present study, where SL Damon on dry enamel (group 7) showed significant lower forces than conventional brackets. Even comparing the results with the study of Sfondrini et al. (2011), there are some differences: in our experimentation, Quick attachments resulted the strongest ones.

However, we have to consider that the different conditions, in which each experimentation takes place, make the comparison between studies very difficult: adhesive system, time between bonding and testing, type of brackets, kind of tooth used, are only few of the elements that can alter the results of each experimentation.

Concerning with saliva contamination, SBS we recorded for each saliva group, were lower than SBS of the corresponding dry group.

The presence of saliva, in fact, negatively influences the bond strength of conventional brackets, because it deposits an organic adhesive coating within the first few sec of exposure that is resistant to washing and causes alterations to the material properties and the substrate surface (Retamoso et al., 2009; Webster et al., 2001; Cacciafesta et al., 2003; Hobson et al., 2001). In those situations in which moisture contamination is expected, the use of other kind of adhesive, such as self-etching or hydrophilic primers, can be suggested (Faltermeier et al., 2007; Cacciafesta et al., 2003; Campoy et al., 2005).

In literature there are no studies that tested saliva contamination on bonding of self-ligating brackets. In this research, the presence of saliva reduced SBS of self-ligating brackets, but this decrease was not statistically significant. Self-ligating brackets resulted less influenced, than conventional attachments, by saliva contamination. This is probably due to different design in mesh pad of bracket bases.

Comparing the different SL brackets tested on contaminated enamel, quick showed the highest values, followed by Smart Clip attachments that presented intermediate bond strengths. Damon brackets, instead, exhibited statistically lower SBS values than all other attachments tested. The base of Quick brackets is provided with wide grooves which allow improvement of composite resin penetration compared with mesh bases, assuring greater bond strengths (Merone et al., 2010).

The analysis of ARI scores reports that with conventional and SL Smart Clip brackets, bond failure, both on dry and contaminated enamel, occurs within the resin, with more than $50 \%$ of adhesive remaining on tooth surface (ARI “2”), while for SL Quick and
Damon, in presence of saliva, there is a reduction of the amount of resin left on the enamel.

In $42,5 \%$ of dry samples, detachment occurred at the adhesive-bracket base interface (ARI "3") while for the most of saliva specimens $(48,7 \%)$ ARI score of " 2 "was recorded, meaning that bond failure happened within the resin. Therefore a reduction of the amount of adhesive left on tooth surface has been detected with the decrease of the values of SBSs, in presence of saliva contamination, when self-ligating brackets are employed in association with conventional acid etching technique and composite resin.

In the present study, neither fractures nor enamel cracks have been noticed.

\section{CONCLUSION}

- Saliva contamination had a negative effect on bond strength provided with conventional acid etching technique and composite resin

- Mean shear bond strength for all groups of brackets tested was over the minimum required for clinical use

- Saliva contamination decreased shear bond strength of conventional brackets significantly, but the reduction of bond strength for self-ligating brackets was not statistically significant

- Both on dry and saliva contaminated enamel, quick SL brackets showed significantly higher SBSs than all other brackets

- ARI scores in contaminated group resulted significantly lower than in dry group

\section{REFERENCES}

Artun, J. and S. Bergland, 1984. Clinical trials with crystal growth conditioning as an alternative to acid-etch enamel pretreatment. Am. J. Orthod., 85: 333-340. PMID: 6231863

Basudan, A.M. and S.E. Al-Emran, 2001. The effects of in-office reconditioning on the morphology of slots and bases of stainless steel brackets and on the shear/peel bond strength. J. Orthod., 28: 231-236. PMID: 11504901

Bishara, S.E., V.V. Gordan, L. VonWald and M.E. Olson, 1998. Effect of an acidic primer on shear bond strength of orthodontic brackets. Am. J. Orthod. Dentofacial Orthop., 114: 243-247. PMID: 9743128

Bishara, S.E., J.F. Laffoon, L. VonWald and J. Warren, 2002. Effect of time on the shear bond strength of cyanoacrylate and composite orthodontic adhesives. Am. J. Orthod. Dentofacial Orthop., 121: 297-300. PMID: 11941344 
Cacciafesta, V., M.F. Sfondrini, M.D. Angelis, A. Scribante and C. Klersy, 2003. Effect of water and saliva contamination on shear bond strength of brackets bonded with conventional, hydrophilic and self-etching primers. Am. J. Orthod. Dentofacial Orthop., 123: 633-640. PMID: 12806342

Cacciafesta, V., M.F. Sfondrini, A. Scribante, M.D. Angelis and C. Klersy, 2004. Effects of blood contamination on the shear bond strengths of conventional and hydrophilic primers. Am. J. Orthod. Dentofacial Orthop., 126: 207-212. DOI: 10.1016/j.ajodo.2003.06.022

Campoy, M.D., A. Vicente and L.A. Bravo, 2005. Effect of saliva contamination on the shear bond strength of orthodontic brackets bonded with a selfetching primer. Angle Orthod., 75: 865-869.

Faltermeier, A., M. Behr, M. Rosentritt, C. Reicheneder and D. Mubig, 2007. An in vitro comparative assessment of different enamel contaminants during bracket bonding. Eur. J. Orthod., 29: 559563. DOI: 10.1093/ejo/cjm052

Farrokhgisu, E. and A. Eskandarizade, 2010. Shear bond strength of four cariostatic tooth colored materials to dentin of permanent premolar teeth. Curr. Res. Dent., 1: 11-14. DOI: 10.3844/crdsp.2010.11.14

Hobson, R.S., J. Ledvinka and J.G. Meechan, 2001. The effect of moisture and blood contamination on bond strength of a new orthodontic bonding material. Am. J. Orthod. Dentofacial Orthop., 120: 54-57. DOI: 10.1067/mod.2001.115037

Jobalia, S.B., R.M. Valente, W.G.D. Rijk, E.A. BeGole and C.A. Evans, 1997. Bond strength of visible light-cured glass ionomer orthodontic cement. Am. J. Orthod. Dentofacial Orthop., 112: 205-208. DOI: 10.1016/S0889-5406(97)70247-6

Knox, J., P. Hubsch, M.L. Jones and J. Middleton, 2000. The influence of bracket base design on the strength of the bracket-cement interface. J. Orthodontics, 27: 249-254.

Merone, G., R. Valletta, R.D. Santis, L. Ambrosio and R. Martina, 2010. A novel bracket base design: biomechanical stability. Eur. J. Orthod., 32: 219223. DOI: $10.1093 /$ jo/cjp077

Millett, D.T., D. Cattanach, R. McFadzean, J. Pattison and J. McColl, 1999. Laboratory evaluation of a compomer and a resin-modified glass ionomer cement for orthodontic bonding. Angle Orthod., 69: 58-63. PMID: 10022186

Newman, G.V., R.A. Newman, B.I. Sun, J.L. Ha and S.A. Ozsoylu, 1995. Adhesion promoters, their effect on the bond strength of metal brackets. Am. J. Orthod. Craniofacial Orthop., 108: 237-241. PMID: 7661138
Northrup, R.G., D.W. Berzins, T.G. Bradley and W. Schuckit, 2007. Shear bond strength comparison between two orthodontic adhesives and selfligating and conventional brackets. Angle Orthod., 77: 701-706. PMID: 17605477

Oztoprak, M.O., F. Isik, K. Sayinsu, T. Arun and B. Aydemir, 2007. Effect of blood and saliva contamination on shear bond strength of brackets bonded with 4 adhesives. Am. J. Orthod. Craniofacial Orthop., 131: 238-242. PMID: 17276865

Paschos, E., J.O. Westphal, N. Ilie, K.C. Huth and R. Hickel et al., 2008. Artificial saliva contamination effects on bond strength of self-etching primers. Angle Orthod., 78: 716-721. PMID: 18302473

Retamoso, L.B., F.M. Collares, E.S. Ferreira and S.M. Samuel, 2009. Shear bond strength of metallic brackets: Influence of saliva contamination. J. Applied Oral, Sci., 17: 190-194. PMID: 19466249

Reynolds, I.R., 1975. A review of direct orthodontic bonding. Br. J. Orthod.., 2: 171-178.

Sfondrini, M.F., V. Cacciafesta, A. Scribante, M.D. Angelis and C. Klersy, 2004. Effect of blood contamination on shear bond strength of brackets bonded with conventional and self-etching primers. Am. J. Orthod. Craniofacial Orthop., 125: 357-360. PMID: 15014415

Sfondrini, M.F., S. Gatti and A. Scribante, 2011. Shear bond strength of self-ligating brackets. Eur. J. Orthod., 33: 71-74. DOI: 10.1093/ejo/cjq041

Sharma-Sayal, S.K., P.E. Rossouw, G.V. Kulkarni and K.C. Titley, 2003. The influence of orthodontic bracket base design on shear bond strength. Am. J. Orthod. Craniofacial Orthop., 124: 74-82. DOI: 10.1016/S0889-5406(03)00311-1

Tavares, S.W., S. Consani, D.F. Nouer, M.B. Magnani and P.R. Nouer et al., 2006. Shear bond strength of new and recycled brackets to enamel. Braz. Dent. J., 17: 44-48. PMID: 16721464

Townsend, R.D. and W.J. Dunn, 2004. The effect of saliva contamination on enamel and dentin using a self-etching adhesive. J. Am. Dent. Assoc., 135: 895-901. PMID: 15354900

Vicente, A., A. Mena, A.J. Ortiz and L.A. Bravo, 2009. Water and saliva contamination effect on shear bond strength of brackets bonded with a moisturetolerant light cure system. Angle Orthod., 79: 127132. PMID: 19123696

Wang, W.N. and T.C. Lu, 1991. Bond strength with various etching times on young permanent teeth. Am. J. Orthod. Dentofacial Orthop., 100: 72-79. DOI: 10.1016/0889-5406(91)70052-X 
Wang, W.N., H.C. Li, T.H. Chou, D.D. Wang and L.H. Lin et al., 2004. Bond strength of various bracket base designs. Am. J. Orthod. Craniofacial Orthop., 125: 65-70. PMID: 14718881

Webster, M.J., R.S. Nanda, M.G. Duncanson, Jr., S.S. Khajotia and P.K. Sinha, 2001. The effect of saliva on shear bond strengths of hydrophilic bonding systems. Am. J. Orthod. Craniofacial Orthop., 119: 54-58. PMID: 11174540
Wright, W.L. and J.M. Powers, 1985. In vitro tensile bond strength of reconditioned brackets. Am. J. Orthod., 87: 247-252. DOI: 10.1016/00029416(85)90046-6 